International Conference Mathematical and Computational Biology 2011

International Journal of Modern Physics: Conference Series

Vol. 9 (2012) 341-346

C World Scientific Publishing Company

DOI: $10.1142 / \mathrm{S} 2010194512005405$

\title{
ON DISCRETE LOTKA-VOLTERRA TYPE MODELS
}

\author{
FARRUKH MUKHAMEDOV \\ Faculty of Science, International Islamic University Malaysia, \\ P.O. Box, 141, 25710, Kuantan, Pahang, Malaysia \\ farrukh_m@iiu.edu.my \\ MANSOOR SABUROV \\ Faculty of Science, International Islamic University Malaysia, \\ P.O. Box, 141, 25710, Kuantan, Pahang, Malaysia \\ msaburov@gmail.com
}

\begin{abstract}
The Lotka-Volterra (in short LV) model is a second order nonlinear differential equation frequently used to describe the dynamics of biological systems in which two groups of species, predators and their preys interact. One of the basic results of the LV model is that under suitable conditions the LV model can exhibit any asymptotical behavior such as equilibrium states, periodic cycles, and attractors. The discrete analogy of LV model has been considered by many researchers and has been called a quadratic LV model. In a discrete case, one of the unexpected results is that a quadratic LV model cannot exhibit a periodic cycle. In this paper we study nonlinear LV type models which include quadratic LV as a particular case. Unlike quadratic LV models, LV type models can exhibit any asymptotical behavior such as equilibrium states, periodic cycles, and attractors.
\end{abstract}

Keywords: LV type model; equilibrium state; periodic cycle.

\section{Look at the Background}

Predator-prey models have been studied for a long time. Many biologists believe that if the unique positive equilibrium point of a predator-prey system is local asymptotically stable, then it is global asymptotically stable. However, this is not always true. It is found that a unique positive local asymptotically stable equilibrium point has at least one limit cycle surrounding the equilibrium point under suitable condition. Therefore, many mathematicians try to use some well-known methods to find conditions for global stability for the equilibrium point of predator-prey systems.

The Lotka-Volterra (in short LV) model is the simplest model of predator-prey interactions. It is based on linear per capita growth rates and written as follows

$$
\left\{\begin{array}{c}
\dot{x}=x(b-p y) \\
\dot{y}=y(-d+r x)
\end{array},\right.
$$


where

- $\quad y$ is the number of some predators (for example, wolves);

- $\quad \boldsymbol{x}$ is the number of its preys (for example, rabbits);

- $\quad \dot{y}$ and $\dot{\boldsymbol{x}}$ represent the growth of the two populations against the time;

- $\quad t$ represents the time;

- $\quad \boldsymbol{b}, \boldsymbol{p}, \boldsymbol{d}, \boldsymbol{r} \geq 0$ are parameters representing the interaction of the two species.

The equation (1) has periodic solutions which do not have a simple expression in terms of the usual trigonometric functions. At any given time in the phase plane, the system is in a limit cycle and lies somewhere on the inside of these elliptical solutions
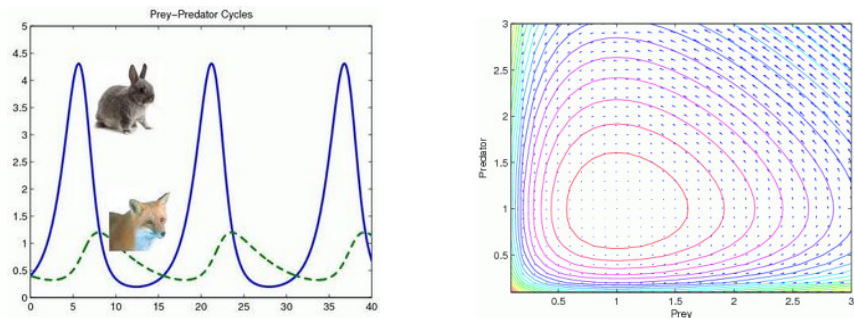

Fig. 1. Periodic activity generated by the LV model

A discrete analogy of the LV model can be considered as follows

$$
\left\{\begin{array} { c } 
{ x _ { n + 1 } - x _ { n } = x _ { n } ( b - p y _ { n } ) } \\
{ y _ { n + 1 } - y _ { n } = y _ { n } ( - d + r x _ { n } ) }
\end{array} \text { or } \left\{\begin{array}{l}
x_{n+1}=x_{n}\left(1+b-p y_{n}\right) \\
y_{n+1}=y_{n}\left(1-d+r x_{n}\right)
\end{array} .\right.\right.
$$

Under suitable conditions, the discrete LV model (2) has an application in genetic population systems. One of the simplest cases of the discrete LV model (2) is the following evolution operator $V: S^{1} \rightarrow S^{1}$ of the population on the one dimensional simplex $S^{1}=\left\{(x, y) \in \mathbb{R}^{2}: x, y \geq 0, x+y=1\right\}$

$$
V:\left\{\begin{array}{l}
x^{\prime}=x(1-a y) \\
y^{\prime}=y(1+a x)
\end{array},\right.
$$

where $\boldsymbol{V}(\boldsymbol{x}, \boldsymbol{y})=\left(\boldsymbol{x}^{\prime}, \boldsymbol{y}^{\prime}\right)$ is an image of the point $(\boldsymbol{x}, \boldsymbol{y})$ under the operator $\boldsymbol{V}$.

In the series of works ${ }^{1,2,3,6}$, a quadratic LV model $\boldsymbol{V}: \boldsymbol{S}^{\boldsymbol{m}-1} \rightarrow \boldsymbol{S}^{\boldsymbol{m}-1}$ defined by

$$
x_{k}^{\prime} \equiv(V x)_{k}=x_{k}\left(1+\sum_{i=1}^{m} a_{k i} x_{i}\right), k=\overline{1, m},
$$

deeply studied on the $(\boldsymbol{m}-1)$ dimensional simplex

$$
S^{m-1}=\left\{x=\left(x_{1}, \cdots, x_{m}\right) \in \mathbb{R}^{m-1}: \sum_{i=1}^{m} x_{i}=1, x_{i} \geq 0, \forall i=\overline{1, m}\right\}
$$

as the generalization of the model (3), where $\boldsymbol{A}_{m}=\left(\boldsymbol{a}_{k i}\right)_{k, i=1}^{m}, \boldsymbol{a}_{k i} \in[-1,1]$ is a skew-symmetric matrix and $\boldsymbol{V} \boldsymbol{x}=\boldsymbol{x}^{\prime}=\left(\boldsymbol{x}_{1}^{\prime}, \cdots, \boldsymbol{x}_{m}^{\prime}\right)$ is an image of the point $\boldsymbol{x} \in \boldsymbol{S}^{m-1}$. 
It is worth mentioning that a quadratic LV model (4) has a fascinating application in genetic population systems ${ }^{4}$. Let us briefly mention it, in order to describe main properties of the quadratic LV model (4).

Consider a population consisting of $\boldsymbol{m}$ species. Let $\boldsymbol{x}=\left(\boldsymbol{x}_{1}, \cdots, \boldsymbol{x}_{\boldsymbol{m}}\right)$ be the probability distribution of species in the initial generations and $\boldsymbol{P}_{i j, k}$ the probability that individuals in the $\boldsymbol{i}$-th and $\boldsymbol{j}$-th species interbreed to produce an individual from $\boldsymbol{k}$-th specie. We assume that in each generation an individual can inherit only species of its parents. In other words, it means that $\boldsymbol{P}_{\boldsymbol{i} j, k}=0, \boldsymbol{k} \notin\{\boldsymbol{i}, \boldsymbol{j}\}$. Then the probability distribution $x^{\prime}=\left(x_{1}^{\prime}, \cdots, x_{m}^{\prime}\right)$ of the species in the next generation can be found by the total probability given by (4), where $\boldsymbol{a}_{\boldsymbol{k} i}=2 \boldsymbol{P}_{\boldsymbol{i}, \boldsymbol{k}}-1$. This means that the association $\boldsymbol{x} \rightarrow \boldsymbol{x}^{\prime}$ defines a mapping $\boldsymbol{V}$ so-called an evolution operator of the population system.

Theorem 1.1 ${ }^{1,2,3,6}$ Suppose the evolution of the population system is given by (4). Then the following statements hold true.

(i) The population system has at least $\boldsymbol{m}$ equilibrium states. The number of equilibrium states is finite. In typical conditions of the nature, equilibrium states are not local asymptotically stable.

(ii) If the population system is at the equilibrium state then it has only an odd number of species. In other words only an odd number of species like one, three, and so forth can survive during the evolution. However, all species would not disappear.

(iii) The population system does not have any periodic cycle.

(iv) If the population system is not at the equilibrium state then in the future, some species of the population system will disappear.

(v) Prehistory of the population system can be uniquely recovered. There exists a unique equilibrium state, from which the history of any state begins. In other words ancestors of any two distinct generations are the same.

(vi) The "end" or "final" state of the evolution is unpredictable. More precisely, the possibility of "end" or "final" states of the evolution is infinite. In other words the future of the evolution is unpredictable.

It seems the picture of the continuous LV model is different from the discrete LV one. For example, in continuous case, a system could have a periodic cycle. In contrast, in discrete case there is not any periodic state. The aim of this paper is that to provide some nonlinear LV type models whose properties different from the properties of the quadratic LV model (4).

\section{Some LV Type Models}

In recent years, a penetration of mathematical model in biological problems is staidly rising. Both continuous and quadratic LV model describe the following evolution law: "LV evolution law"- the absent species of the initial state will not appear during the future evolution. In the series of works ${ }^{5}$ it is obtained an explicit form of all models which are caring an LV evolution law. 
Let $S^{m-1}$ be an $(\boldsymbol{m}-1)$ dimensional simplex defined by (5) and $\boldsymbol{I}_{\boldsymbol{m}}=\{1,2, \cdots, \boldsymbol{m}\}$ be a finite set. The set $\Gamma_{\boldsymbol{\alpha}}=\left\{\boldsymbol{x} \in \boldsymbol{S}^{\boldsymbol{m}-1}: \boldsymbol{x}_{\boldsymbol{k}}=0, \forall \boldsymbol{k} \in \boldsymbol{\alpha}\right\}$ is called a face of the simplex, where $\boldsymbol{\alpha} \subset \boldsymbol{I}_{m}$. The set $\boldsymbol{r i}\left(\Gamma_{\boldsymbol{\alpha}}\right)=\left\{\boldsymbol{x} \in \boldsymbol{S}^{\boldsymbol{m}-1}: \boldsymbol{x}_{\boldsymbol{k}}=0, \forall \boldsymbol{k} \in \boldsymbol{\alpha}\right\}$ is called a relatively interior of $\Gamma_{\boldsymbol{\alpha}}$.

A mapping $V: S^{m-1} \rightarrow S^{m-1}$ is said to be a nonlinear LV type model if it is defined as follows

$$
x_{k}^{\prime}=(V x)_{k}=x_{k}\left(1+f_{k}(x)\right), k=\overline{1, m}, \quad x \in S^{m-1}
$$

where a mapping $\boldsymbol{F}: \boldsymbol{S}^{m-1} \ni \boldsymbol{x} \rightarrow \boldsymbol{F}(\boldsymbol{x}) \equiv\left(f_{1}(\boldsymbol{x}), \cdots, f_{m}(\boldsymbol{x})\right) \in \mathbb{R}^{m}$ satisfies the following conditions

(i) A mapping $\boldsymbol{F}: \boldsymbol{S}^{m-1} \rightarrow \mathbb{R}^{m}$ is continuous;

(ii) For every $x \in S^{m-1}$ one has $f_{k}(x) \geq-1, \forall k=\overline{1, m}$;

(iii) For every $x \in S^{m-1}$ one has $\sum_{k=1}^{m} x_{k} f_{k}(x)=0$;

(iv) For every $\boldsymbol{\alpha} \subset I_{m}$ one has $f_{k}(x)>-1$ for every $\boldsymbol{x} \in \boldsymbol{r i}\left(\Gamma_{\boldsymbol{\alpha}}\right)$ and $\boldsymbol{k} \in \boldsymbol{\alpha}$.

Theorem 2.1 $\mathbf{1}^{5}$ A model $V: S^{m-1} \rightarrow S^{m-1}$ describes an LV evolution law if and only if it is a nonlinear LV type model.

It is worth mentioning that nonlinear LV type models were deeply studied ${ }^{5}$ whenever $^{2}$ a mapping $\boldsymbol{F}: \boldsymbol{S}^{\boldsymbol{m}-1} \rightarrow \mathbb{R}^{m}$ satisfies the following condition

$$
(\boldsymbol{F}(\boldsymbol{x})-\boldsymbol{F}(\boldsymbol{y}), \boldsymbol{x}-\boldsymbol{y}) \geq 0 .
$$

Such kind of nonlinear LV type models are called $\boldsymbol{F}$-monotone LV type models. It is easy to check that a quadratic LV model (4) is a $\boldsymbol{F}$ - monotone LV type model with

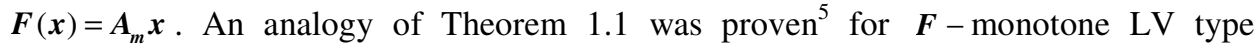
models.

Problem 2.2 What is the meaning of inequality (6) in the population system? Which kind of laws does inequality (6) describe?

In this paper we study a nonlinear LV type model which is not $\boldsymbol{F}$ - monotone.

Let us consider the following nonlinear LV type model $V_{\varepsilon}: S^{m-1} \rightarrow S^{m-1}$

$$
\left(V_{\varepsilon}(x)\right)_{k}=x_{k}\left(1+\varepsilon\left(x_{k}-\sum_{i=1}^{m} x_{i}^{2}\right)\right), k=\overline{1, m},
$$

where, $\boldsymbol{\varepsilon} \in[-1,1] \backslash\{0\}$.

Theorem 2.3 Suppose the LV evolution law of the population system is given by (7). Then the following statements hold true.

(i) The population system has $2^{m}-1$ number of equilibrium states. The population system does not have any equilibrium state except the equally distributed states. All equilibrium states are local as well as global asymptotically stable.

(ii) The future of the evolution is predictable.

(iii) If $\varepsilon>0$ then the species of the initial state dominating in terms of the number will survive forever during the evolution and the rest species will disappear. At the end of the evolution the surviving species will be equally distributed.

(iv) If $\boldsymbol{\varepsilon}<0$ then all involving species of the initial state will survive forever during the evolution and their portion will be equal at the end of the evolution. 
Let us consider the following nonlinear LV type model $\boldsymbol{W}: \boldsymbol{S}^{m-1} \rightarrow \boldsymbol{S}^{m-1}$

$$
(W x)_{k}=x_{k}\left(1+x_{k}^{2}+2 \sum_{i=1}^{k-1} x_{i}-\sum_{i=k}^{m} x_{i}-3 \sum_{\substack{i, j=1 \\ i<j}}^{k-1} x_{i} x_{j}\right) .
$$

Theorem 2.4 If the LV evolution law of the population system is given by (8) then the prehistory of the evolution cannot be recovered. In other words, there are different ancestors who have the same generation.

Suppose one dimensional simplex $S^{1}$ has the following decomposition

$$
\boldsymbol{S}^{1}=\boldsymbol{C}_{1} \cup \boldsymbol{C}_{2} \cup \boldsymbol{C}_{3} \cup \boldsymbol{C}_{4} \cup \boldsymbol{C}_{5}
$$

where $\boldsymbol{C}_{i}=\left\{\left(x_{1}, \boldsymbol{x}_{2}\right)=\boldsymbol{x} \in \boldsymbol{S}^{1}: \boldsymbol{a}_{i} \leq \boldsymbol{x}_{1} \leq \boldsymbol{a}_{i+1}\right\}, \boldsymbol{i}=\overline{1,5} \quad$ and $\quad\left(\boldsymbol{a}_{1}, \cdots, \boldsymbol{a}_{6}\right)=\left(0, \frac{9}{30}, \frac{11}{30}, \frac{19}{30}, \frac{21}{30}, 1\right)$. Let us consider the following nonlinear LV type model $\boldsymbol{V}: \boldsymbol{S}^{1} \rightarrow \boldsymbol{S}^{1}$

$$
\begin{gathered}
\left\{\begin{array}{l}
(V x)_{1}=x_{1}\left(1+f_{1}\left(x_{1}, x_{2}\right)\right) \\
(V x)_{2}=x_{2}\left(1+f_{2}\left(x_{1}, x_{2}\right)\right)
\end{array},\left\{\begin{array}{ll}
2 & x \in C_{1} \\
\frac{9}{10 x_{1}}-1, & x \in C_{2} \\
\frac{4 x_{2}-2}{x_{1}} & x \in \boldsymbol{C}_{3} \\
\frac{1}{10 x_{1}}-1 & x \in \boldsymbol{C}_{4} \\
-\frac{2 x_{2}}{x_{1}} & x \in C_{5}
\end{array}, f_{2}\left(x_{1}, x_{2}\right)=\left\{\begin{array}{cc}
-\frac{2 x_{1}}{x_{2}} & x \in C_{1} \\
\frac{1}{10 x_{2}}-1, & x \in C_{2} \\
\frac{4 x_{1}-2}{x_{2}} & x \in C_{3} \\
\frac{9}{10 x_{2}}-1 & x \in C_{4} \\
2 & x \in C_{5}
\end{array} .\right.\right.\right.
\end{gathered}
$$

Theorem 2.5 If the LV evolution law of the population system is given by (9) then the population system has periodic cycles.

\section{Conclusions}

The LV model is the simplest model of predator-prey interactions. One of the basic results of the LV model is that under suitable conditions the LV model can exhibit any asymptotical behavior such as equilibrium states, periodic cycles, and attractors. It seems that the picture of the continuous LV model is different from the discrete LV one. For example, in continuous case, a system could have a periodic cycle. In contrast, in discrete case there is not any periodic state. In this paper we provide some nonlinear LV type models whose properties different from the properties of the quadratic LV model (4). However, the provided models have the same properties as the classical LV model have an asymptotical behavior.

\section{Acknowledgments}

The Authors acknowledge Research Endowment Grant B (EDW B 0905-303) of IIUM and the MOSTI grant 01-01-08-SF0079. 


\section{References}

1. R.N. Ganikhodzhaev, Rus. Acad. Sci. Sbor. Math., 76, 489-506 (1993).

2. R.N. Ganikhodzhaev, Mathematical Notes, 56, 1125-1131 (1994).

3. R.N. Ganikhodzhaev and D. Eshmamatova, Vladikavkaz, Math. Jour. 8, 2-13 (2006).

4. Yu. Lyubich, Mathematical structures in population genetics, (Springer-Verlag, Berlin, 1992).

5. R.N. Ganikhodzhaev and M. Saburov, J. Sib. Fed. Math \& Phys. 2, 188-196 (2008).

6. R.N. Ganikhodzhaev, F. Mukhamedov and U. Rozikov, Quadratic stochastic operators and processes: Results and open problems, Infin. Dim. Anal., Quantum Prob. and Related Topics 14 (2) 279-335 (2011). 